\title{
Spectroscopic observations of the $\delta$ Scorpii binary during its recent periastron passage ${ }^{\star}$
}

\author{
A. S. Miroshnichenko ${ }^{1,2}$, J. Fabregat ${ }^{3}$, K. S. Bjorkman ${ }^{1}$, D. C. Knauth ${ }^{1}$, N. D. Morrison ${ }^{1}$, A. E. Tarasov ${ }^{4}$, \\ P. Reig ${ }^{5,6}$, I. Negueruela ${ }^{7}$, and P. Blay ${ }^{3}$ \\ 1 Ritter Observatory, Dept. of Physics \& Astronomy, University of Toledo, Toledo, OH 43606, USA \\ 2 Central Astronomical Observatory of the Russian Academy of Sciences at Pulkovo, 196140 Saint-Petersburg, \\ Russia \\ 3 Universidad de Valencia, Departamento de Astronomía, 46100 Burjassot, Valencia, Spain \\ 4 Crimean Astrophysical Observatory and Isaac Newton Institute of Chile, Crimean Branch, Nauchny, Crimea, \\ 98409, Ukraine \\ ${ }^{5}$ Foundation for Research and Technology-Hellas, 71110 Heraklion, Crete, Greece \\ 6 Physics Department, University of Crete, 71033 Heraklion, Crete, Greece \\ 7 Observatoire de Strasbourg, 11 rue de l'Université, 67000 Strasbourg, France
}

Received 16 March 2001 / Accepted 20 June 2001

\begin{abstract}
The bright star $\delta$ Sco has been considered a typical B0-type object for many years. Spectra of the star published prior to 1990 showed no evidence of emission, but only of short-term line profile variations attributed to nonradial pulsations. Speckle interferometric observations show that $\delta$ Sco is a binary system with a highlyeccentric orbit and a period of $\sim 10.6$ years. Weak emission in the $\mathrm{H} \alpha$ line was detected in its spectrum for the first time during a periastron passage in 1990. Shortly before the next periastron passage in the summer of 2000, the binary entered a strong $\mathrm{H} \alpha$ emission and enhanced mass-loss phase. We monitored the spectroscopic development of the Be outburst from July 2000 through March 2001. In this paper we present results from our spectroscopy, refine elements of the binary orbit, and discuss possible mechanisms for the mass loss.
\end{abstract}

Key words. stars: emission-line, Be - binaries: close - individual: $\delta$ Sco - techniques: spectroscopic

\section{Introduction}

The bright $(V=2.3 \mathrm{mag})$ B0.3IV star $\delta$ Scorpii (HD 143275, HR 5953) has been considered a typical B0-type object for many years. It was suspected of binarity nearly a century ago by Innes (1901), who claimed visual detection of the secondary during a lunar occultation. van Hoof et al. (1963) found its radial velocity $(R V)$ to vary with an amplitude of $\sim 15 \mathrm{~km} \mathrm{~s}^{-1}$ on a time scale of 20 days. Their data were obtained in February 1955 and showed a smooth, nearly sinusoidal, variation over the period of the observations. Based on 7 observations obtained in 5 different nights in 1974 and 1976, Levato et al. (1987) also detected $R V$ variations with even a larger amplitude of $\sim 25 \mathrm{~km} \mathrm{~s}^{-1}$ and suggested a period of 83 days. These

Send offprint requests to: A. S. Miroshnichenko,

e-mail: anatoly@physics.utoledo.edu

* This paper is partly based on observations obtained at the European Southern Observatory and the South African Astronomical Observatory. facts might imply that $\delta$ Sco is a single-lined spectroscopic binary, and such $R V$ variations are easily detectable with modern high-resolution spectrographs, similar to those we used for this study. Below we further discuss this problem.

Smith (1986) performed high-resolution spectroscopic observations of the Si III 4552-4574 $\AA$ lines and found short-term line profile variability. He attributed this phenomenon to nonradial pulsations and treated the star as single due to the absence of mass transfer.

Long-term speckle interferometric observations of $\delta$ Sco (Bedding 1993; Hartkopf et al. 1996) determined that it is a binary system with a highly-eccentric orbit. The orbital parameters found by these authors are significantly different even though they used almost the same data sets (see Table 1). Hartkopf et al. (1996) noticed that the positional residuals tend to decrease as the eccentricity approaches 1 . Their orbital solution predicts very large radial velocity $(R V)$ variations near periastron for both components, with the line peak separation reaching $150 \mathrm{~km} \mathrm{~s}^{-1}$. The components' brightness ratio is estimated 
Table 1. Orbital solutions for $\delta$ Sco.

\begin{tabular}{llllllll}
\hline$T_{0}$ & $a$ & $e$ & $P$ & $\omega$ & $\Omega$ & $i$ & Ref. \\
& $\operatorname{arcsec}$ & & years & degrees & degrees & degrees \\
\hline 1979.3 & 0.11 & 0.82 & 10.5 & 170 & 0 & 70 & 1 \\
$1979.41 \pm 0.14$ & $0.107 \pm 0.007$ & $0.92 \pm 0.02$ & $10.58 \pm 0.08$ & $24 \pm 13$ & $159.3 \pm 7.6$ & $48.5 \pm 6.6$ & 2 \\
\hline $2000.693 \pm 0.008$ & $0.107^{\mathrm{a}}$ & $0.94 \pm 0.01$ & $10.58^{\mathrm{a}}$ & $-1 \pm 5$ & 175 & $38 \pm 5$ & 3 \\
\hline
\end{tabular}

$T_{0}$ is the periastron passage epoch, $a$ is the orbital semi-major axis, $e$ is the orbit eccentricity, $P$ is the orbital period, $\omega$ is the periastron longitude, $\Omega$ is the node line longitude, $i$ is the orbital inclination angle. References: 1 - Bedding (1993), 2 - Hartkopf et al. (1996), 3 - this work.

a - The parameter is taken from the Hartkopf et al. (1996) solution.

to be 1.5-2.0 mag based on an optical interferometric observation obtained at the Anglo-Australian Telescope on 1991 May 31/June 1 (Bedding 1993).

Analysing profiles of $\mathrm{Si}$ III and $\mathrm{Si}$ IV ultraviolet lines, Snow (1981) calculated a mass loss rate of $\sim 3 \times$ $10^{-11} M_{\odot} \mathrm{yr}^{-1}$ from the star. This value is the lowest one among those obtained with the same technique for the stars of his sample, which mainly contained Be stars.

Coté \& van Kerkwijk (1993), who were searching for unidentified Be stars, displayed an $\mathrm{H} \alpha$ line profile of $\delta$ Sco obtained at ESO in 1990 (close to the predicted periastron passage), which showed a weak double-peaked emission component inside a broad photospheric absorption. Previous high-resolution spectroscopic data obtained by Heasley \& Wolff (1983), apparently in 1981/2 at CFHT, and by Grigsby et al. (1992) in 1986 at KPNO showed no emission component in $\mathrm{H} \alpha$.

The HIPPARCOS parallax of the star is $8.12 \pm$ 0.88 milliarcsec (ESA 1997), which corresponds to a distance $D=123 \pm 15$ pc. Other important parameters are $v \sin i=148 \pm 8 \mathrm{~km} \mathrm{~s}^{-1}$ (Brown \& Verschueren 1997), $E_{B-V}=0.14 \mathrm{mag}$, and $\log L_{\mathrm{bol}} / L_{\odot}=4.4 \pm 0.1$ (with the contribution of the secondary subtracted). The star's effective temperature as determined by different methods resulted in slightly different values: $T_{\text {eff }}=29760 \mathrm{~K}$ (Blackwell, Petford, \& Shallis 1980, infrared flux method) and $27500 \mathrm{~K}$ (Heasley et al. 1982, model atmospheres). Grigsby et al. (1992) fitted profiles of several hydrogen and helium lines to non-LTE, line-blanketed model atmospheres and concluded that the fits for $T_{\text {eff }}=27000 \mathrm{~K}$ and $28000 \mathrm{~K}(\log g=4.0$ in both cases $)$ are almost equally good for $\delta$ Sco. The infrared flux method is less accurate because it involves the bolometric stellar flux, which is poorly known for such a hot star due to the lack of farUV observations (see Hummer et al. 1988).

In June 2000, S. Otero discovered a brightening of $\delta$ Sco by visual comparison with nearby 1 st and 2 nd magnitude stars. This information along with the results of first spectroscopic observations, showing the $\mathrm{H} \alpha$ line in emission, was reported by Fabregat et al. (2000) in late July. Since that time the star was monitored by visual and photoelectric photometry as well as by spectroscopy (our team) until it became inaccessible in November. The photometric data have been recently published by
Otero et al. (2001). The results of our spectroscopic observations are presented in this paper.

Our observations are described in Sect. 2, characteristics of the detected spectral lines in Sect. 3, refinement of the orbital solution by means of the RV measurements in Sect. 4, a brief discussion of possible mechanisms responsible for the appearance of the Be phenomenon in $\delta$ Sco in Sect. 5. Detailed modelling of the observed event is beyond the scope of this paper and will be presented elsewhere.

\section{Observations and data reduction}

High-resolution spectroscopic observations of $\delta$ Sco were obtained at the $1 \mathrm{~m}$ telescope of Ritter Observatory (Toledo, OH, USA) between 2000 August 5 and 2001 February 20, at the $2.6 \mathrm{~m}$ Shajn telescope of the Crimean Astrophysical Observatory (CAO, Ukraine) between 2000 July 29 and 2000 August 21, and at $1.52 \mathrm{~m}$ telescope at the ESO (La Silla, Chile) on 2000 October 23. Low-resolution spectroscopic observations were performed with the $1.3 \mathrm{~m}$ telescope of the Skinakas Observatory (Crete, Greece) on 2000 July 19-22 and with the $1.52 \mathrm{~m}$ G. D. Cassini telescope at the Loiano Observatory (BOL, Italy) 2000 July 26 and 29. We also found two archived, previously unpublished, spectra. One was obtained at Ritter on 1994 May 18 in almost the same spectral region and with the same equipment as in 2000. The other one was obtained at the $1.9 \mathrm{~m}$ telescope of the South African Astronomical Observatory on 1998 April 16 with the GIRAFFE fiber-fed échelle spectrograph, which gives a resolving power $R \simeq 39000$ in the range 5200-10400 $\AA$.

At Ritter we used a fiber-fed échelle spectrograph with a Wright Instruments Ltd. CCD camera. The spectra consisted of 9 non-overlapping $\sim 70 \AA$ orders in the range 5285-6597 $\AA$ with $R \simeq 26000$. The Ritter data were reduced with IRAF $^{1}$. In total 24 spectra were obtained; the exposure time for each spectrum was $20 \mathrm{~min}$. Most of the spectra have a signal-to-noise ratio $(S / N) \geq 100$. The following lines were detected: He I 5876, Si II $6347 \& 6371 \AA$, $\mathrm{H} \alpha$ (emission), and Na I $\mathrm{D}_{1,2} 5889 \& 5895 \AA$ (absorption).

\footnotetext{
${ }^{1}$ IRAF is distributed by the National Optical Astronomy Observatories, which are operated by the Association of Universities for Research in Astronomy, Inc., under contract with the National Science Foundation.
} 
At CAO the Coude spectrograph with a GEC CCDdetector $(576 \times 380$ pixel array $)$ was used. All observations were obtained in the second order of a diffraction grating with a reciprocal dispersion of $3 \AA \mathrm{mm}^{-1}$, which corresponds to $R \sim 40000$. A typical exposure time for each spectrum was $4-10 \mathrm{~min}$, giving a $S / N \geq 200$. A region of $31 \AA$ around the $\mathrm{H} \alpha$ (5 spectra) and He I $6678 \AA$ line (4 spectra) was covered in all cases. Data reduction was done with the SPE code developed by S. G. Sergeev at $\mathrm{CAO}$.

At ESO the Fiber-fed Extended Range Optical Spectrograph (FEROS) and a thinned back-illuminated EEV detector with $2048 \times 409615-\mu \mathrm{m}$ pixels were used. The spectrum was obtained in the range $3700-9000 \AA$ with $R \simeq 48000$ and a $S / N \simeq 120$ near $\mathrm{H} \alpha$; the exposure time was $3 \mathrm{~min}$. The spectrum was reduced with the FEROS data reduction package under MIDAS.

At Skinakas the telescope was equipped with a $2000 \times 800$ ISA SITe chip CCD, a 1301 line $\mathrm{mm}^{-1}$ grating, and an $80 \mu \mathrm{m}$ width slit, giving a dispersion of $1 \AA$ pixel $^{-1}$ $(R \simeq 3000)$. The range $5500-7550 \AA$ was observed on July 19-20, while the range $3750-5750 \AA$ was observed on July 21-22. Ten spectra with exposure times from 1 to $5 \mathrm{~s}$ were obtained.

At BOL the telescope was equipped with the Bologna Faint Object Spectrograph and Camera (BFOSC) and several gratings $(R \simeq 1000-2000$ in the range 5400-8100 A). On July 29 the \#9 grism in échelle mode was used with the grism \#10 as cross-disperser, which gives $R \simeq 6000$ in the region 3692-8046 $\AA$. Both the Skinakas and BOL spectra were reduced using the Starlink supported FIGARO package (Shortridge et al. 1997).

The log of our observations is presented in Table 2. Due to the northern location of all the observing sites, the star was observed at large airmasses, and strong telluric water vapour lines appear in the spectra. Because of the high resolution of our data, most of these lines are unblended. They were removed by interpolation between unaffected spectral regions, which were used for construction of the normalized line profiles.

\section{Results}

The Ritter observation obtained in 1994 revealed a weak emission in the $\mathrm{H} \alpha$ line similar to that detected by Coté \& van Kerkwijk (1993) in 1990. The line profile is shown in Fig. 1a along with a theoretical one, calculated with the code SYNSPEC (Hubeny et al. 1995) for the atmospheric parameters from Heasley et al. (1982), the rotational velocity from Brown \& Verschueren (1997), and the solar chemical composition. The equivalent width $(E W)$ of the emission part (after the theoretical profile subtraction) is $0.24 \AA$, while the peak separation is $350 \mathrm{~km} \mathrm{~s}^{-1}$. The emission component in the SAAO 1998 spectrum is hardly recognizable.

The presence of an emission component in the He I $5876 \AA$ line is dubious in 1994 and is not seen in 1998 (see Fig. 1b). Its absorption component is deeper than the
Table 2. Summary of spectroscopic observations of $\delta$ Sco in 2000-2001.

\begin{tabular}{|c|c|c|c|c|}
\hline $\begin{array}{l}\text { Date } \\
2000\end{array}$ & $\begin{array}{c}\text { HJD } \\
2451000+\end{array}$ & Obs. & $\begin{array}{l}\text { Sp.region } \\
\AA\end{array}$ & $n$ \\
\hline $07 / 19$ & 745.264 & Ski & $5500-7550$ & 2 \\
\hline $07 / 20$ & 746.302 & Ski & $5500-7550$ & 3 \\
\hline $07 / 21$ & 747.323 & Ski & $3750-5750$ & 2 \\
\hline $07 / 22$ & 748.302 & Ski & $3750-5750$ & 3 \\
\hline $07^{\prime} / 25$ & 751.313 & BOL & $5400-8100$ & 3 \\
\hline $07 / 28$ & 754.286 & $\mathrm{CAO}$ & $\mathrm{H} \alpha$ & 1 \\
\hline $07 / 29$ & 755.320 & BOL & $3692-8046$ & 1 \\
\hline $08 / 04$ & 761.575 & Rit & $5290-6600$ & 1 \\
\hline 08/07 & 764.246 & $\mathrm{CAO}$ & $\mathrm{H} \alpha$ & 1 \\
\hline $08 / 07$ & 764.568 & Rit & $5290-6600$ & 1 \\
\hline $08 / 09$ & 766.239 & $\mathrm{CAO}$ & He I 6678 & 1 \\
\hline 08/09 & 766.258 & $\mathrm{CAO}$ & $\mathrm{H} \alpha$ & 1 \\
\hline $3 / 10$ & 767.592 & Rit & $5290-6600$ & 1 \\
\hline $3 / 12$ & 769.246 & $\mathrm{CAO}$ & He I 6678 & 1 \\
\hline $3 / 12$ & 769.269 & $\mathrm{CAO}$ & $\mathrm{H} \alpha$ & 1 \\
\hline $3 / 13$ & 770.207 & $\mathrm{CAO}$ & Не I 6678 & 1 \\
\hline $08 / 20$ & 777.225 & $\mathrm{CAO}$ & $\mathrm{H} \alpha$ & 1 \\
\hline $08 / 20$ & 777.233 & $\mathrm{CAO}$ & Не г 6678 & 1 \\
\hline$/ 20$ & 777.546 & Rit & $5290-6600$ & 1 \\
\hline$/ 24$ & 781.577 & Rit & 529 & 1 \\
\hline $08 / 30$ & 787.566 & Rit & 529 & 2 \\
\hline $08 / 31$ & 88.548 & Rit & 5290 & 2 \\
\hline $09 / 04$ & 792.538 & Rit & 5290 & 1 \\
\hline & 794.541 & Rit & 529 & 1 \\
\hline$/ 12$ & 800.527 & Rit & 5290 & 1 \\
\hline & 801.525 & Rit & 529 & 1 \\
\hline & 04.532 & Rit & 529 & 1 \\
\hline $09 / 17$ & 805.524 & Rit & 5290 & 1 \\
\hline & 806.508 & Rit & 5290 & 1 \\
\hline$/ 19$ & 807.506 & Rit & 5290 & 1 \\
\hline $09 / 21$ & 809.506 & Rit & 5290 & 1 \\
\hline & 814.494 & Rit & 5290 & 1 \\
\hline & 815.502 & Rit & 5290 & 1 \\
\hline $09 / 28$ & 816.503 & Rit & $5290-6600$ & 1 \\
\hline & 821.497 & Rit & 5290 & 1 \\
\hline $10 / 23$ & 841.493 & $\mathrm{ESO}$ & $3700-9000$ & 1 \\
\hline $02 / 10^{\mathrm{a}}$ & 951.948 & Rit & $5290-6600$ & 1 \\
\hline $02 / 20^{\mathrm{a}}$ & 961.904 & Rit & $5290-6600$ & \\
\hline $02 / 27^{\mathrm{a}}$ & 968.978 & Rit & $5290-6600$ & 1 \\
\hline $03 / 09^{\mathrm{a}}$ & 978.944 & Rit & $5290-6600$ & 1 \\
\hline
\end{tabular}

The spectral range in Col. 4 expressed either in $\AA$ or by the spectral line in its centre.

Multiple spectra obtained during the same date (Col. 5) were co-added.

a The spectrum was obtained in 2001 .

theoretical one for the described atmospheric parameters, as was noticed by Heasley et al. (1982) for other early B-type stars (including $\delta$ Sco). This difference might also be due to the presence of a shell component or to an effect of microturbulence (see Leone \& Lanzafame 1998). In fact, the He I line $E W$ in the 1994 and 1998 spectra is almost the same as the value reported by Heasley et al. (1982), $0.73 \pm 0.01 \AA$ vs. $0.69 \AA$. Thus, there is no evidence for a growth in the amount of circumstellar matter around $\delta$ Sco during a significant portion of the last binary cycle.

All our spectra of $\delta$ Sco obtained during the 2000/1 campaign contain emission lines. All emission lines detected in our high-resolution spectra have doublepeaked profiles (Fig. 2) with a mean separation of the blue and red peak of $250 \mathrm{~km} \mathrm{~s}^{-1}$ (He I and Si II lines) 

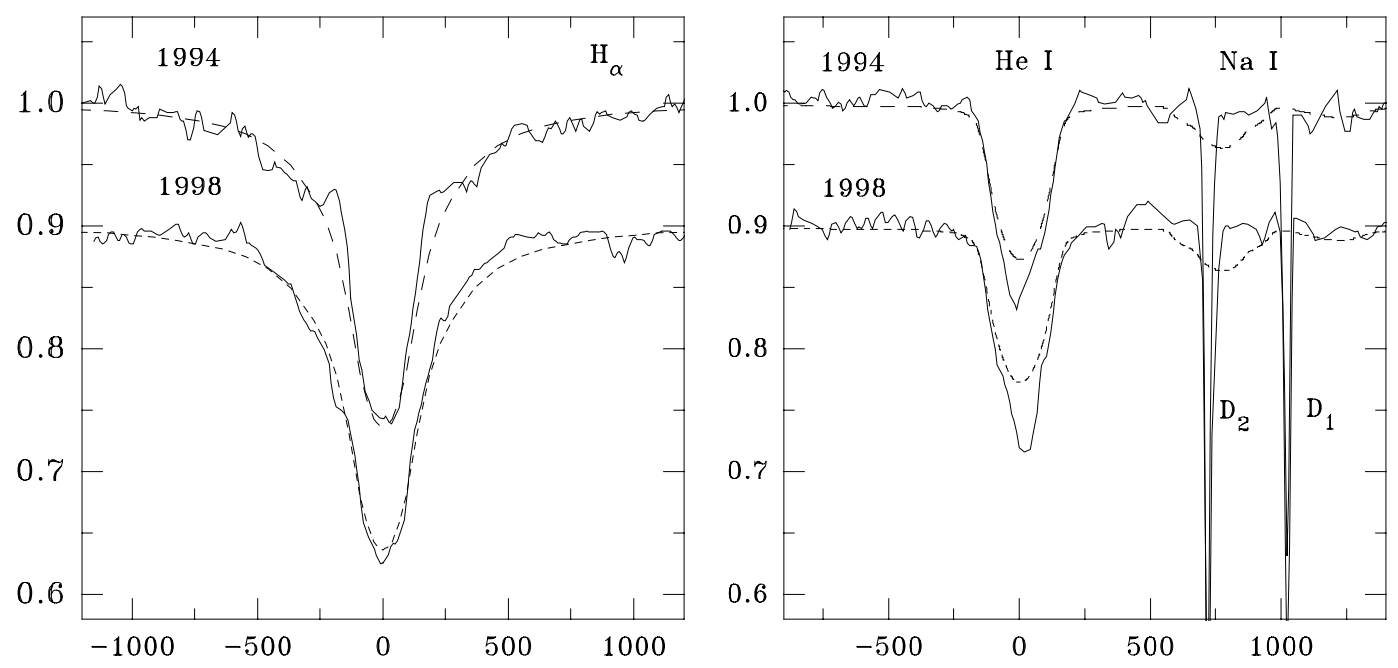

Fig. 1. The H $\alpha$ and He I $5876 \AA$ lines in the 1994 Ritter and the 1998 SAAO spectra. The observational data are shown by solid lines, while the theoretical profiles for $T_{\text {eff }}=27500 \mathrm{~K}, \log g=4.0$, and $v \sin i=150 \mathrm{~km} \mathrm{~s}^{-1}$ are shown by dashed lines. The intensity scale is normalized to the level of the underlying continuum, while the heliocentric $R V$ are given in $\mathrm{km} \mathrm{s}^{-1}$.

and $150 \mathrm{~km} \mathrm{~s}^{-1}(\mathrm{H} \alpha)$. Such a profile shape suggests that the lines are formed in a circumstellar disk, typical for classical Be stars. The peak separation difference can be explained by the fact that the He and Si lines are formed closer to the star, where the disk rotates faster.

The normalized $\mathrm{H} \alpha$ profiles obtained at CAO are systematically weaker and narrower than those obtained at Ritter. This might be due to the narrower spectral region observed at CAO, which contains a small area useful for continuum determination, and a stronger contamination of the CAO data by the telluric lines. However, this does not affect the $R V \mathrm{~s}$ and has no impact on our conclusions. Parameters of the $\mathrm{H} \alpha$ line profiles are presented in Table 3.

Our ESO spectrum covers the whole optical range and contains information about both photospheric and circumstellar features. The double-peaked emission is present in all hydrogen lines up to $\mathrm{H}_{8}$ and in most of the He I lines (see Fig. 3). Other species detected (e.g., O II, He II, N II, $\mathrm{N}$ III, Si IV) show typical photospheric absorption profiles, which are consistent with the mentioned fundamental parameters. No clear signs of the secondary were found in the spectrum. This might imply that the components' temperature difference is not large enough to produce additional detectable spectral features. However, this suggestion needs to be verified by higher-resolution future observations.

Our results obtained for the $\mathrm{H} \alpha$, He I $5876 \AA$, and Si II 6347 and $6371 \AA$ lines showed that their intensities were increasing until September 12 and decreasing after that time. The $\mathrm{H} \alpha$ line $F W H M$ displays a similar behaviour. The $E W$ s of all 4 mentioned lines showed anticorrelation with the visual brightness of the system. Their $R V \mathrm{~s}$, measured by matching the direct and reversed line profiles, were shifting to the blue when the system was fading and vice versa (Figs. 2a,b and 4). At the same time, the He I $6678 \AA$ line exhibited a small variability of the peak strengths between August 9 and 13, but was not detected at all on August 20 (Fig. 2c). No sign of the Fe II $5317 \AA$ line was detected in the 2000 Ritter spectra. It appeared first in the ESO spectrum and is seen in the 2001 Ritter spectra with a larger strength (Fig. 2d). Other Fe II lines, most of which have weak emission components, are also seen in the ESO spectrum. The Na I D lines are purely interstellar in origin and exhibit no change in strengths or positions.

\section{Refining the binary orbit}

$\delta$ Sco has been observed with the speckle interferometric technique at several observatories for a long time (1973-1991). In total $\sim 30$ observations were obtained. Most of the data are collected in McAlister \& Hartkopf (1988), later observations were published by Bedding (1993), McAlister et al. (1990), and Fu et al. (1997). The data analysis has led to two different orbital solutions presented by Bedding (1993) and Hartkopf et al. (1996). As one can see in Fig. 5, the data show a large scatter, suggesting that the orbital solution needs to be tested by other observational techniques. One of the options is spectroscopy, which gives information about the components' $R V \mathrm{~s}$.

We were unable to measure $R V \mathrm{~s}$ of the photospheric lines close to periastron (except for those in the ESO spectrum). However, the circumstellar matter (where the emission lines originate) moves with the star and should reflect the star's motion. Indeed, the orbital motion of a Be star was detected using $R V$ s of the $\mathrm{H} \alpha$ emission line in such binaries as $\phi$ Per (Božić et al. 1995) and $\gamma$ Cas (Harmanec et al. 2000). Thus we think it reasonable to use our $R V$ measurements to constrain the orbital solution for $\delta$ Sco. The strongest line in the spectrum, and the one that gives the most reliable $R V \mathrm{~s}$, is $\mathrm{H} \alpha$. We estimate 
Table 3. Characteristics of the $\mathrm{H} \alpha$ line in the spectra of $\delta$ Sco.

\begin{tabular}{|c|c|c|c|c|c|c|c|c|c|}
\hline \multirow{2}{*}{$\begin{array}{l}\text { Date } \\
2000\end{array}$} & \multirow{2}{*}{$\begin{array}{c}\text { HJD } \\
2451000+\end{array}$} & \multirow[t]{2}{*}{ Obs. } & \multicolumn{7}{|c|}{$\mathrm{H} \alpha$} \\
\hline & & & $\begin{array}{c}E W \\
\AA\end{array}$ & $I_{\mathrm{b}}$ & $\overline{I_{\mathrm{r}}}$ & $\overline{I_{\mathrm{c}}}$ & $\begin{array}{c}R V_{\text {mean }} \\
\mathrm{km} \mathrm{s}^{-1}\end{array}$ & $\begin{array}{l}\Delta V_{\text {peak }} \\
\mathrm{km} \mathrm{s}^{-1}\end{array}$ & $\begin{array}{l}F W H M \\
\mathrm{~km} \mathrm{~s}^{-1}\end{array}$ \\
\hline $07 / 19$ & 745.264 & Ski & 2.92 & 1.41 & 1.40 & & & & \\
\hline $07 / 20$ & 746.302 & Ski & 3.15 & 1.41 & 1.41 & & & & \\
\hline $07 / 25$ & 751.313 & BOL & 3.00 & 1.35 & & & & & \\
\hline $07 / 28$ & 754.286 & $\mathrm{CAO}$ & 2.59 & 1.46 & 1.41 & 1.30 & -17.0 & 150 & 272 \\
\hline $07 / 29$ & 755.320 & BOL & 3.40 & 1.42 & & & & & \\
\hline $08 / 04$ & 761.575 & Rit & 3.37 & 1.55 & 1.53 & 1.39 & -19.5 & 152 & 295 \\
\hline $08 / 07$ & 764.246 & $\mathrm{CAO}$ & 2.64 & 1.46 & 1.45 & 1.33 & -18.5 & 151 & 283 \\
\hline $08 / 07$ & 764.568 & Rit & 3.28 & 1.55 & 1.52 & 1.37 & -21.0 & 155 & 293 \\
\hline 08/09 & 766.258 & $\mathrm{CAO}$ & 2.76 & 1.51 & 1.47 & 1.33 & -19.5 & 154 & 277 \\
\hline $08 / 10$ & 767.592 & Rit & 3.59 & 1.57 & 1.55 & 1.39 & -19.5 & 154 & 298 \\
\hline $08 / 12$ & 769.269 & $\mathrm{CAO}$ & 2.99 & 1.52 & 1.49 & 1.37 & -25.0 & 152 & 278 \\
\hline $08 / 20$ & 777.225 & $\mathrm{CAO}$ & 3.03 & 1.50 & 1.53 & 1.37 & -28.0 & 154 & 278 \\
\hline $08 / 20$ & 777.546 & Rit & 4.18 & 1.61 & 1.64 & 1.46 & -28.5 & 151 & 305 \\
\hline $08 / 24$ & 781.577 & Rit & 4.35 & 1.64 & 1.62 & 1.47 & -30.0 & 153 & 309 \\
\hline $08 / 30$ & 787.566 & Rit: & 4.62 & 1.65 & 1.60 & 1.50 & -35.0 & & \\
\hline $08 / 31$ & 788.548 & Rit & 4.68 & 1.64 & 1.63 & 1.54 & -38.5 & 165 & 333 \\
\hline $09 / 04$ & 792.538 & Rit & 5.16 & 1.73 & 1.67 & 1.57 & -48.5 & 160 & 321 \\
\hline 09/06 & 794.541 & Rit & 5.01 & 1.72 & 1.71 & 1.57 & -48.0 & 160 & 321 \\
\hline $09 / 12$ & 800.527 & Rit & 5.26 & 1.77 & 1.69 & 1.57 & -54.0 & 160 & 320 \\
\hline 09/13 & 801.525 & Rit & 4.98 & 1.71 & 1.68 & 1.56 & -49.0 & 155 & 320 \\
\hline 09/16 & 804.532 & Rit & 4.99 & 1.69 & 1.68 & 1.57 & -46.0 & 155 & 323 \\
\hline 09/17 & 805.524 & Rit & 4.82 & 1.67 & 1.68 & 1.56 & -40.5 & 155 & 321 \\
\hline 09/18 & 806.508 & Rit & 4.90 & 1.68 & 1.68 & 1.55 & -38.5 & 150 & 324 \\
\hline 09/19 & 807.506 & Rit & 4.60 & 1.71 & 1.61 & 1.55 & -41.5 & 152 & 316 \\
\hline $09 / 21$ & 809.506 & Rit & 4.82 & 1.73 & 1.66 & 1.60 & -33.5 & 150 & 308 \\
\hline $09 / 26$ & 814.494 & Rit & 4.06 & 1.65 & 1.59 & 1.49 & -30.5 & 145 & 292 \\
\hline $09 / 27$ & 815.502 & Rit & 3.89 & 1.63 & 1.54 & 1.49 & -31.0 & 150 & 292 \\
\hline $09 / 28$ & 816.503 & Rit & 3.90 & 1.64 & 1.59 & 1.48 & -26.0 & 145 & 283 \\
\hline $10 / 03$ & 821.497 & Rit & 3.32 & 1.62 & 1.50 & 1.44 & -24.5 & 145 & 271 \\
\hline $10 / 23$ & 841.493 & ESO & 2.75 & 1.48 & 1.49 & 1.37 & -18.0 & 150 & 275 \\
\hline $02 / 10^{\mathrm{a}}$ & 951.948 & Rit & 3.80 & 1.59 & 1.59 & 1.47 & -8.5 & 135 & 282 \\
\hline $02 / 20^{\mathrm{a}}$ & 961.904 & Rit & 4.00 & 1.60 & 1.61 & 1.49 & -8.0 & 135 & 282 \\
\hline $02 / 27^{\mathrm{a}}$ & 968.978 & Rit & 4.00 & 1.61 & 1.62 & 1.49 & -7.5 & 135 & 282 \\
\hline $03 / 10^{\mathrm{a}}$ & 978.944 & Rit & 4.34 & 1.60 & 1.65 & 1.51 & -7.5 & 130 & 300 \\
\hline
\end{tabular}

The intensities of the blue and red emission peaks normalized to the underlying continuum are listed in Cols. 5 and 6 , respectively; the intensity of the central depression is given in Col. 7; the $R V$ of the overall profile is given in Col. 8; the peak separation and full width at half-maximum are given in Cols. 9 and 10, respectively.

The low-resolution Skinakas data are not suitable for the $R V$ measurements. The BOL data do not resolve the double-peaked $\mathrm{H} \alpha$ structure.

The Ritter data marked with a colon have the lowest $S / N$ ratio.

${ }^{a}$ The spectrum was obtained in 2001.

the mean accuracy of the individual $\mathrm{H} \alpha R V \mathrm{~s}$ to be about $1-2 \mathrm{~km} \mathrm{~s}^{-1}$.

We suggest that this line is formed in the disk around the primary component, and its mean $R V$ coincides with that of the star. This suggestion seems to be fairly justified, since the line profile has almost equal peak strengths $(V / R \simeq 1)$. The latter indicates an almost axisymmetric matter distribution in the disk. Moreover, most of the nearly symmetric absorption lines in the ESO spectrum that allow reliable $R V$ measurements (such as Si III 4552, He I 4143, absoprtion wings of $\mathrm{H} \beta$, and some others) gave values within $\pm 2 \mathrm{kms}^{-1}$ of that from the $\mathrm{H} \alpha$ line.

In order to compare the measured $R V \mathrm{~s}$ with predictions from the orbital solutions, one needs to know the systemic velocity $\left(v_{\text {sys }}\right)$. The $R V$ measurements for $\delta$ Sco published so far span a range from -26 to $+6 \mathrm{~km} \mathrm{~s}^{-1}$. Since the orbital eccentricity is large, the $R V \mathrm{~s}$ obtained within approximately a year around a periastron passage are 

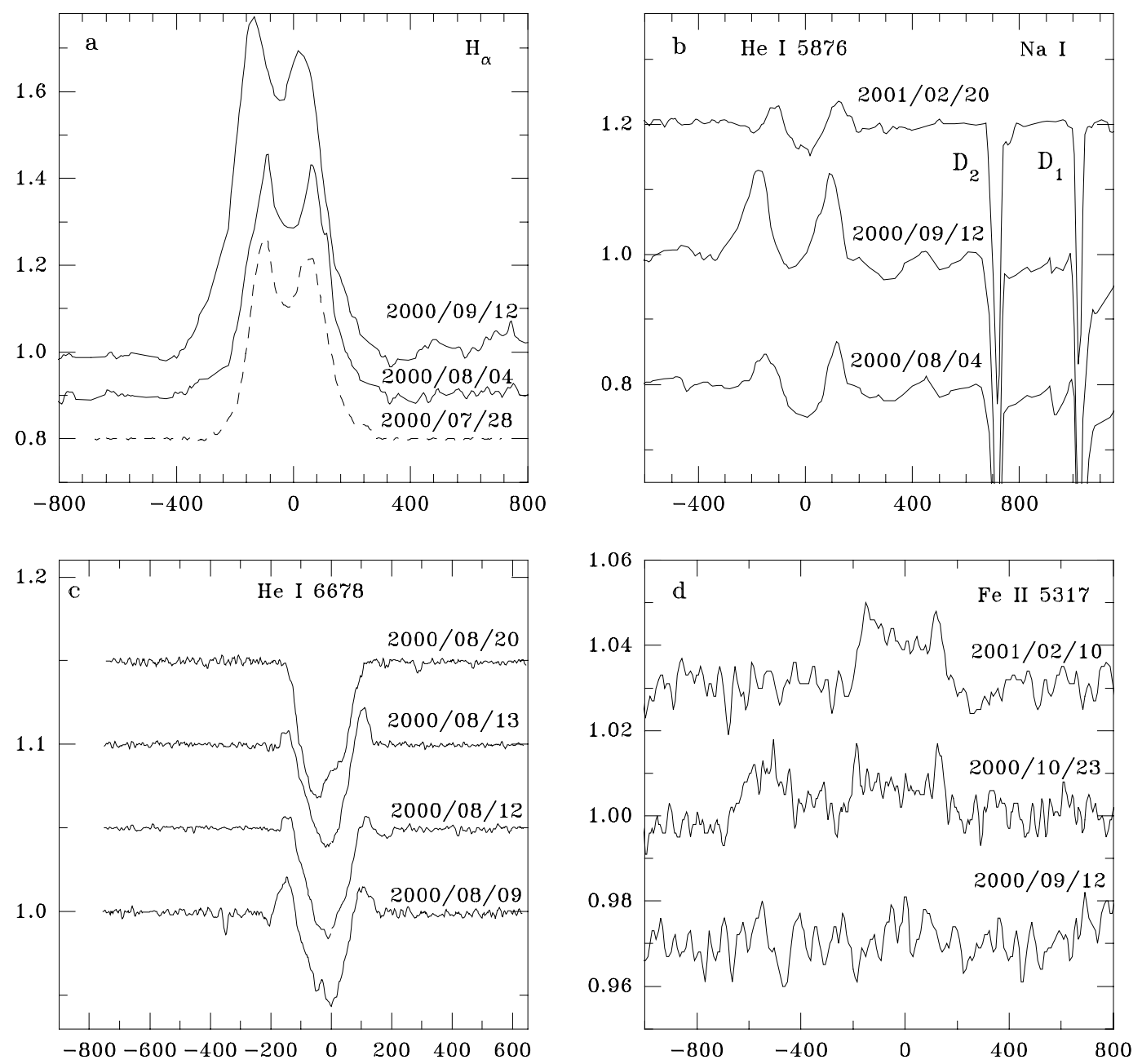

Fig. 2. The emission line profiles of $\delta$ Sco. a) The $\mathrm{H} \alpha$ profiles with extreme RVs obtained at Ritter (solid lines). The CAO $\mathrm{H} \alpha$ profile obtained on 2000 July 28 is shown by a dash-dotted line. b) The He I $5876 \AA$ line profiles obtained at Ritter. c) The He I $6678 \AA$ line profiles obtained at CAO. d) Time evolution of the Fe II $5317 \AA$ line. The intensity and heliocentric $R V$ are given in the same units as in Fig. 1.

affected by the components' acceleration and cannot be adopted as a systemic velocity. Analysis of the literature data show that the most accurate value, which is probably not affected by the periastron acceleration, is $-7 \mathrm{~km} \mathrm{~s}^{-1}$ (Evans 1967). This value turned out to be in good agreement with our independent estimate from the orbital solution fitting described below as well as with that derived from several absorption lines in the 1998 SAAO spectrum $\left(-5 \mathrm{~km} \mathrm{~s}^{-1}\right)$.

According to our data, the $\mathrm{H} \alpha R V$ minimum took place between 2000 September 7 and 12 (see Table 3 ). This is about 5 months later than the Bedding (1993) prediction for the periastron passage and about 1.5 months later than the Hartkopf et al. (1996) prediction. Furthermore, both previous orbital solutions predicted a noticeably longer $R V$ minimum (see Fig. 6).

Our observations turned out to provide constraints for most of the orbital parameters. The time coverage constrains the periastron epoch $\left(T_{0}\right)$ within several days. The symmetry of the $R V$ curve suggests that the perias- tron longitude $(\omega)$ is close to 0 , while its depth depends on the components' mass ratio $(q)$, the eccentricity $(e)$, and the orbital inclination angle $(i)$. At the same time, $e, i$, and the node line longitude $(\Omega)$ affect the orbit spatial orientation, while $\Omega$ has no impact on the RV curve. The eccentricity also controls the width of the $R V$ curve around periastron. The mass ratio $(q \sim 1.5-2.0$ depending on the secondary's spectral type) was estimated by Bedding (1993) using the brightness ratio mentioned in Sect. 1. The central depression depth of the $\mathrm{H} \alpha$ profiles indicates that the disk inclination angle is intermediate $\left(\sim 30^{\circ}-50^{\circ}\right)$. However, we should note here that line profile shapes do not always give unambiguous information about the disk inclination angle (e.g., Quirrenbach et al. 1997). We assume that the disk plane coincides with the orbital plane, and the primary's rotational axis is perpendicular to this plane. At least, we do not have any evidence that the disk is tilted.

Having in hand our $R V$ data and the speckle interferometry information, we calculated the $R V$ curve and 

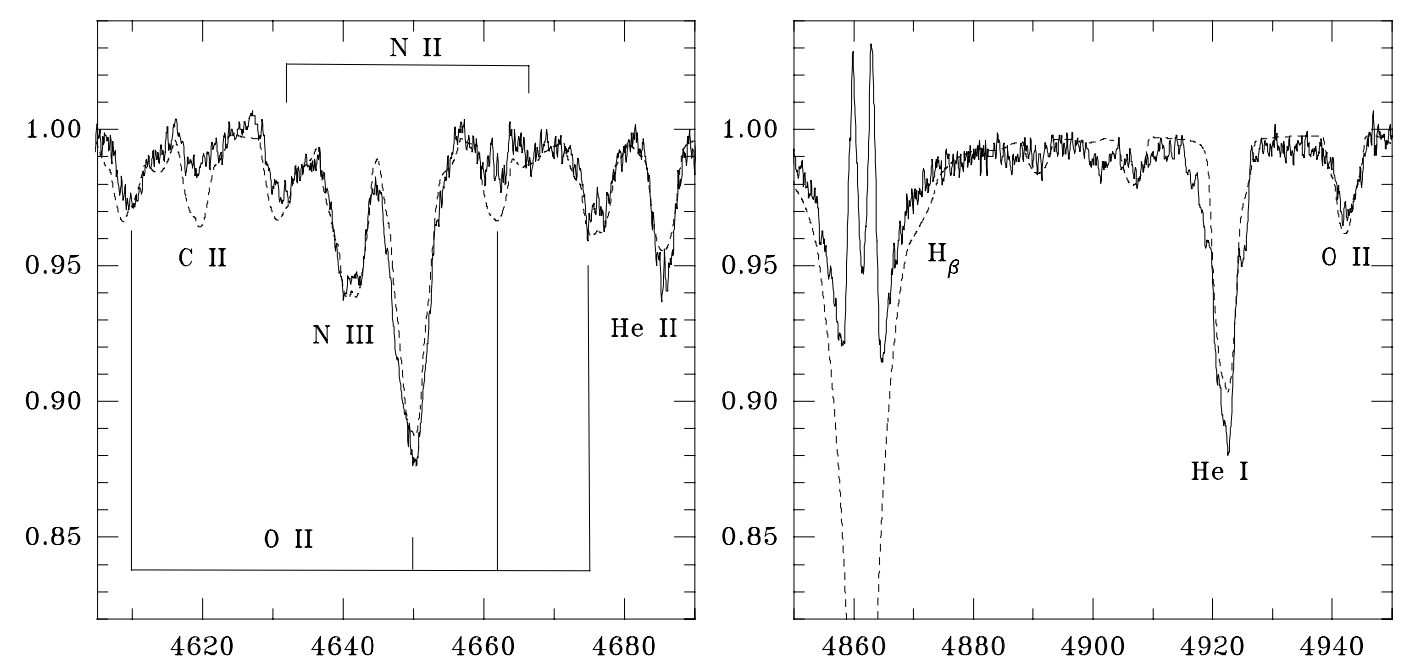

Fig. 3. Portions of the ESO spectrum of $\delta$ Sco obtained on 2000 October 23. The observational data are shown by a solid line, while the theoretical profiles for $T_{\text {eff }}=27000 \mathrm{~K}, \log g=4.0$, and $v \sin i=150 \mathrm{~km} \mathrm{~s}^{-1}$ are shown a dashed line. The intensity is normalized to the underlying continuum level, while the heliocentric wavelengths are given in $\AA$.

the orbit projection in the plane of the sky for different sets of the orbital parameters. The best solution is listed in the last line of Table 1 . It is based on a study of the 6 parameters $\left(e, i, \omega, T_{0}, q\right.$, and $\left.v_{\text {sys }}\right)$ space, which also provided us with the uncertainty estimates. This study showed that $i$ and $q$ are not independent in the vicinity of the best fit. Their relationship can be expressed as $i=(15.8 \pm 0.3) q+(10.6 \pm 0.5)$ and gives $i=42^{\circ}$ for $q=2.0$ and $i=34^{\circ}$ for $q=1.5$. The best value of $v_{\text {sys }}$ is $-6 \pm 0.5 \mathrm{~km} \mathrm{~s}^{-1}$. It coincides with the mean $R V$ we measured using the 1998 SAAO spectrum taking into account $\mathrm{a}+1 \mathrm{~km} \mathrm{~s}^{-1}$ correction, which follows from our orbital solution. There are two orbital parameters we cannot estimate from our data: the orbital period and the semi-major axis. We adopt them from the Hartkopf et al. (1996) solution, since its predicted $R V$ curve is closer to the observed one than that of the Bedding (1993) solution.

Thus, our spectroscopic data improve the orbital solution for the $\delta$ Sco system. The solution is now consistent with both the $R V$ and interferometric data.

\section{Discussion}

Our new orbital solution confirms the conclusion, drawn earlier on the basis of the interferometric data, that $\delta$ Sco is a binary system in a high-eccentricity orbit. The presence of an additional stellar component, which may be responsible for the short-term $R V$ variations reported by van Hoof et al. (1963) and Levato et al. (1987), would result in a more complicated $R V$ curve than the observed one. The short-term $R V$ amplitude is well explained by the periastron acceleration. Perturbations in the velocity curve near periastron due to a third body would be easily detected in the Ritter data due to their high accuracy and dense coverage around this orbital phase.

Another important and not easily resolvable problem concerns the mass loss and the disk formation mechanism. All the spectroscopic data available show that the emission-line spectrum of the primary was stronger during the current (2000) periastron passage than during the previous one. This suggests that the amount of circumstellar matter, and hence the mass loss, grows with time. One possibility to induce the mass loss is the mass transfer through the inner Lagrangian point $L_{1}$, if at least one of the components fills its Roche lobe. According to our orbital solution, the smallest distance between the stars at periastron is $d=0.06 a$, where $a$ is the orbital semimajor axis. Using the components' mass ratio and the rotational parameter of the system $F_{1}=0.12$ (the ratio of the primary's angular rotation velocity and the mean orbital angular velocity), one can calculate that the equilibrium point at periastron is located at $x_{L_{1}}=0.61 d$ from the primary's center (Kallrath \& Milone 1998). This point has the same meaning as $L_{1}$ for close binaries in circular orbits. Since $d \sim 24 R_{1}$, where $R_{1}$ is the primary's radius calculated from the bolometric luminosity and the spectroscopic $T_{\text {eff }}$ presented in Sect. 1 , and $x_{L_{1}}=14.6 R_{1}$, the primary is unlikely to fill its Roche lobe during a relatively fast periastron passage. The situation is almost the same for the secondary, which is supposed to be even smaller than the primary.

The presence of a cool secondary, filling its Roche lobe, can be ruled out by the following arguments. As we will show below, the line emission appeared before the optical brightening, while one would expect the opposite in the case of the Roche lobe filling by the secondary. Furthermore, it is easy to show that even at periastron, when an upper limit for the secondary's radius is $\sim 10 R_{1}$, the system would brighten by $\geq 0.7 \mathrm{mag}$ in the $V$-band if the secondary's $T_{\text {eff }} \geq 5000 \mathrm{~K}$. If the secondary is cooler, then the overall brightness increase would be smaller, but the absorption-line spectrum of the secondary would be easily detectable. None of these phenomena was observed. Thus, direct mass transfer is not expected in the present binary orbit. 


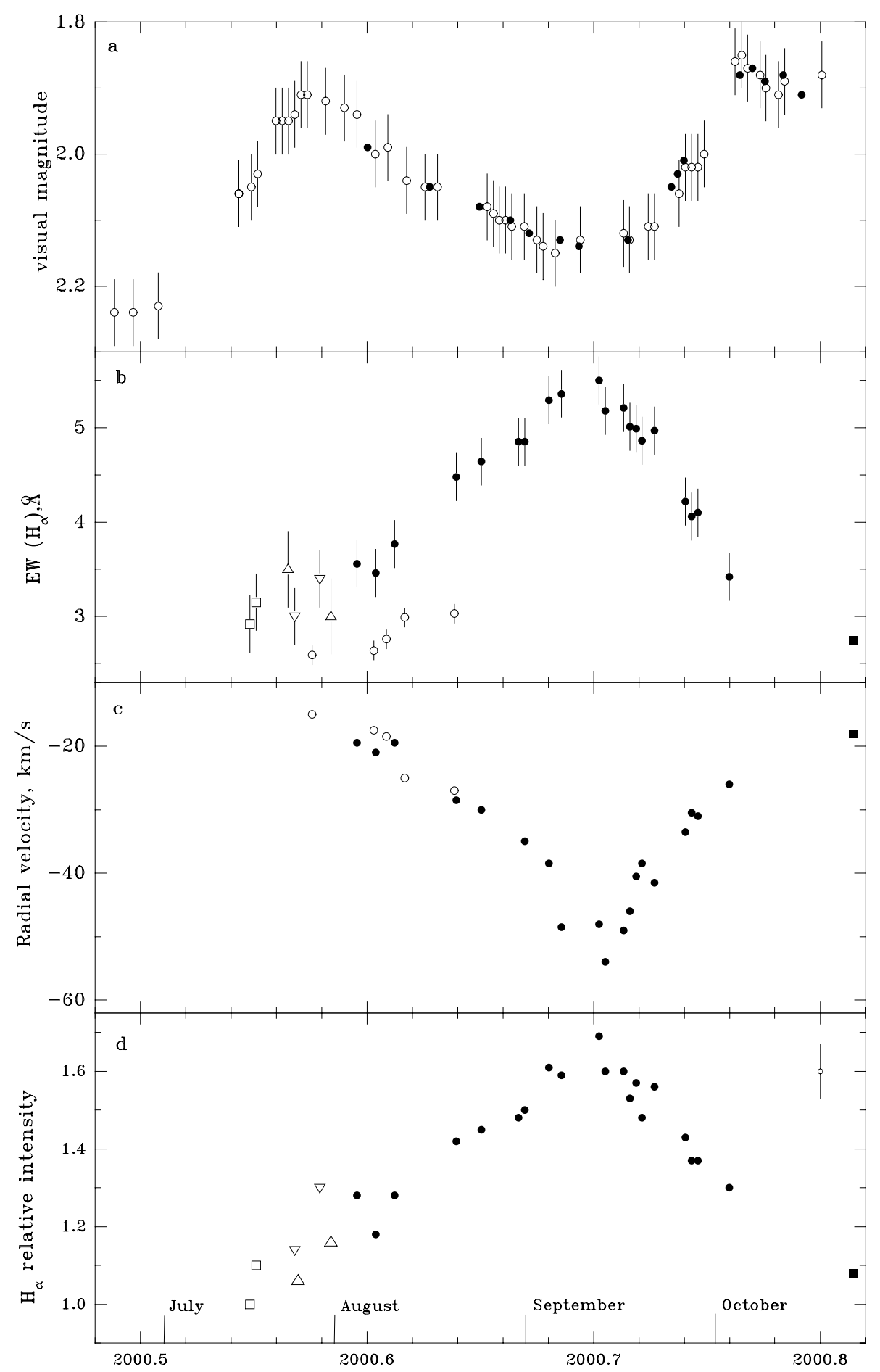

Fig. 4. The photometric and spectroscopic variations of $\delta$ Sco during 2000. a) The light curve from Otero et al. (2001). The visual observations are shown by open circles, and the photoelectric data by filled circles. The variation of the $H \alpha E W \mathrm{~s}, R V \mathrm{~s}$ (from Table 3), and relative flux are shown in panels b), c), and $\mathbf{d}$ ), respectively. The Skinakas data are shown by open squares, those obtained by French amateurs by open upward triangles, the BOL data by open downward triangles, the CAO data by open circles, the Ritter data by filled circles, and the ESO data by filled squares. Uncertainties of the $R V$ s are comparable with the symbol size, while that of the intensities is shown in the upper right-hand corner of panel $\mathbf{d}$ ).

As mentioned above, Smith (1986) found $\delta$ Sco to show nonradial pulsations, which may result in mass loss. However, the UV spectra (Snow 1981) indicate that the mass loss from the star is extremely small. Nevertheless, $\delta$ Sco displays a detectable emission-line spectrum starting from 1990. The data from Coté \& van Kerkwijk (1993) and our campaign show that the emission was observed near periastron, and that there was no noticeable increase 
of the emission between the last 2 periastron passages. Hence, one may argue that the emission strength increase correlates with the binary period and with the periastron passage time in particular. Since the components are very close to each other at periastron, tidal interaction between them may become significant enough to amplify the primary's pulsational instability and trigger an enhanced mass loss. This is a working hypothesis which needs to be verified by modelling. Alternatively, an unrelated growth of the disk mass (such as is seen in other Be stars) may have been coincidentally underway at the time of this periastron passage.

Let us now study the combined photometric and spectroscopic behaviour of $\delta$ Sco. The photometric data obtained before 1990 reveal an almost constant visual brightness, $V=2.32 \pm 0.01 \mathrm{mag}$. The only different result $(V=$ $2.21 \mathrm{mag}$ ) was published by Hogg (1958). However, comparison of his photometry $\left(V_{\mathrm{H}}\right)$ with the modern Johnson system data $\left(V_{\mathrm{J}}\right)$ shows that the colour difference was not properly taken into account in Hogg's catalogue. For example, for blue stars $V_{\mathrm{H}} \leq V_{\mathrm{J}}$, while for red stars the situation is reversed. As a result, Hogg's measurement does not differ from other values within the inter-system translation errors.

Between 1990 January 22 and 1992 July 24 the star was monitored by the HIPPARCOS satellite (ESA 1997). This period covered the previous periastron passage, and the data show no noticeable variations. The mean brightness registered by HIPPARCOS, $\bar{V}=2.29 \pm 0.01 \mathrm{mag}$ (translated into the Johnson system using a formula by Harmanec 1998), is very close to the normal brightness of the star. The HIPPARCOS value is 3 per cent brighter which is not significant due to statistical errors of the translation. Thus, there is no evidence for any photometric variation of $\delta$ Sco before the year 2000 .

The 2000 photometric observations began visually on June 26 (Otero et al. 2001). By July 4 the star was marginally brighter than usual $\left(m_{\mathrm{vis}}=2.24 \mathrm{mag}\right)$. The first spectroscopic observations of $\delta$ Sco known to us in 2000 were obtained by the French amateurs D. and S. Morata ${ }^{2}$ on June 2. They show that the $\mathrm{H} \alpha$ line was nearly as strong as in our first spectra. Between July 16 and 28 the star brightened up to $m_{\text {vis }}=1.9 \mathrm{mag}$ and then experienced a $\sim 2$-month brightness minimum (hereafter referred to as "the dip"), which coincided in time with the $\mathrm{H} \alpha E W$ and intensity maximum and the line $R V$ minimum (see Fig. 4). The dip ended in the beginning of October, and the brightness remained stable at $m_{\text {vis }}=1.89 \pm 0.02 \mathrm{mag}$ until October 18 , when the star became inaccessible. The new observing season started in late December at a similar brightness level. Although the results of visual observers differ up to 30 per cent, the mean star's magnitude through March 2001 is $m_{\mathrm{vis}}=$ $1.95 \pm 0.15$.

\footnotetext{
2 data accessible at

http://perso. wanadoo.fr/sdmorata/pages/7DelSco.html
}

Analysing the described variations, we emphasize the following facts:

1. The photometric dip and $\mathrm{H} \alpha E W$ and $F W H M$ maxima occurred simultaneously and were both centered at the minimum $R V$, i.e. at periastron;

2. The $\mathrm{H} \alpha$ line integrated flux also reached its maximum at periastron;

3. The $\mathrm{H} \alpha$ peak separation slightly increased towards periastron and gradually decreased afterwards.

All these phenomena can be explained in the framework of the following scenario. The system consists of a B0-type primary, surrounded by a circumstellar gaseous disk, and a more compact secondary. The system is not eclipsing, since the orbit is far from an edge-on orientation. Before periastron the disk began to form (or to grow) and kept growing with time. The rise of the $\mathrm{H} \alpha$ intensity during the dip suggests that the amount of matter in the disk was increasing, which, in turn, suggests an increase of the disk's optical depth (at least before periastron).

The disk's growing size and optical depth resulted in two major effects. The disk contribution to the overall system brightness through free-free emission and a partial attenuation of the primary's surface both increased with time. An interplay between these factors was responsible for the dip's shape. In June 2000 the disk was apparently small. It produced the $\mathrm{H} \alpha$ emission, while its continuum emission was roughly compensated by the attenuation so that the overall brightening of the system was small. This also suggests that the starting point of the observed enhanced mass loss took place not long before the first spectroscopic observation of $\delta$ Sco in 2000 .

In July the disk emission grew faster than the attenuation. At some threshold point, the decreasing separation between the stellar components limited the disk's ability to grow further before periastron because it reaches the primary's Roche lobe size. This implied a density increase on the side of the disk facing the secondary which, however, smeared out quickly because of the disk's rotation, with a period of a few days. The disk's enhanced density caused its larger optical depth, which resulted in the overall brightness fading. After periastron the components' separation began to increase, allowing the disk to grow freely again. As a result, the disk's density decreased, and the dip ended. The disk's density variations can be seen in Fig. 7, where larger values of the relative peak separation correspond to larger densities (see Hanuschik et al. 1988). It is also seen that the density dropped noticeably 5 months after periastron (triangles in Fig. 7). Thus, a combination of the primary's attenuation and the disk optical brightness, which depends on both its optical depth and its spatial extent, is capable of a qualitative explanation of the dip occurrence.

This scenario explains most details of the system behaviour in 2000. The observed behaviour of $\delta$ Sco during the last binary cycle suggests that close periastron passages play an important role in the mass loss process 


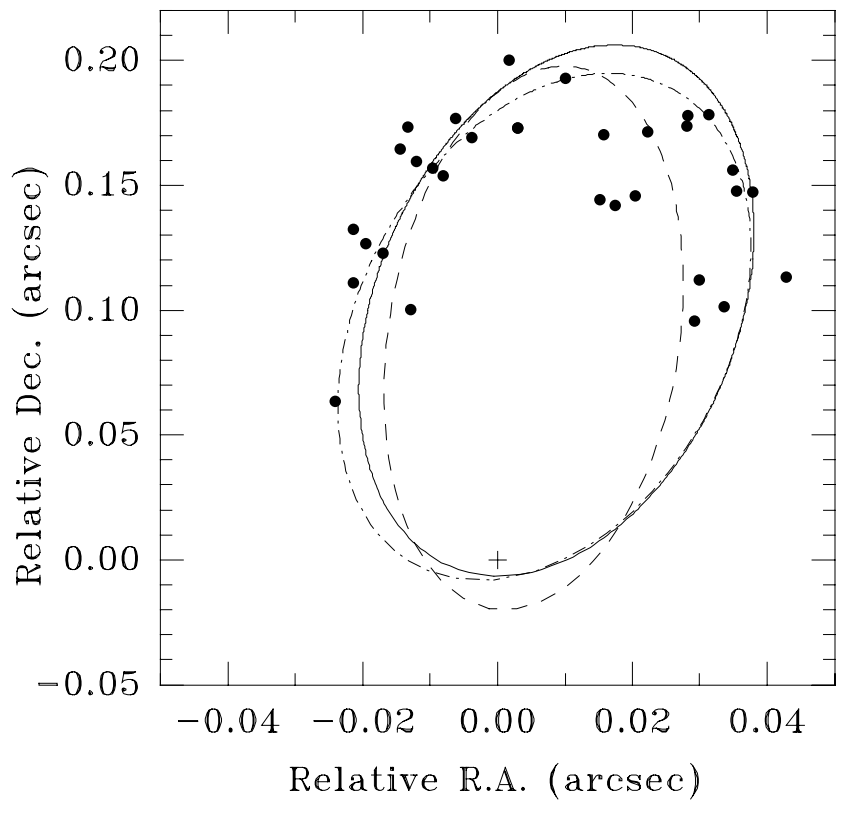

Fig. 5. The binary orbit in the plane of the sky. The speckle interferometry data are shown by filled circles. The primary component is placed at the origin of the coordinate system, and is marked with a cross. The dashed line represents the orbital solution from Bedding (1993), the dashed-dotted line shows the solution from Hartkopf et al. (1996), and the solid line shows our solution.

observed in this binary system. However, there are a number of problems to be solved. These include the mass loss mechanism, the nature of the secondary, and the temporal evolution of the Fe II $5317 \AA$ line (see Fig. 2d). They will require follow up observations and modelling.

The overall behaviour of $\delta$ Sco during this period of outburst provides a chance to see the onset of the emission line activity, which can reveal fundamental information about the building of stellar envelopes and the causes of the Be phenomenon. In this sense, it is interesting to compare the present $\delta$ Sco episode with the onset of activity in other Be stars. One remarkable and well-studied example is $\mu$ Cen, which in the 1980's presented several activity episodes arising from a quiescent status characterized by an absorption-line spectrum (Peters 1986; Hanuschik et al. 1993). A first comparison shows that the time scales involved are very different. Outbursts in $\mu$ Cen had a fast rise lasting 2-10 days, and a slower decay during one to two months. Conversely, the current $\delta$ Sco outburst is still in the rising phase (by June 2001), one year after the first detected activity. The smooth increase of emission line strength and visual magnitude in $\delta$ Sco is much more like the onset of activity in $\gamma$ Cas in the 1950's after several years of quiescence, leading to its current active phase which has lasted more than 50 years by now (Cowley \& Marlborough 1968; Doazan et al. 1983). $\mu$ Cen also experienced a long lived outburst which started in 1989 and led into an active phase lasting to the present time. Superimposed on this long term active phase, several

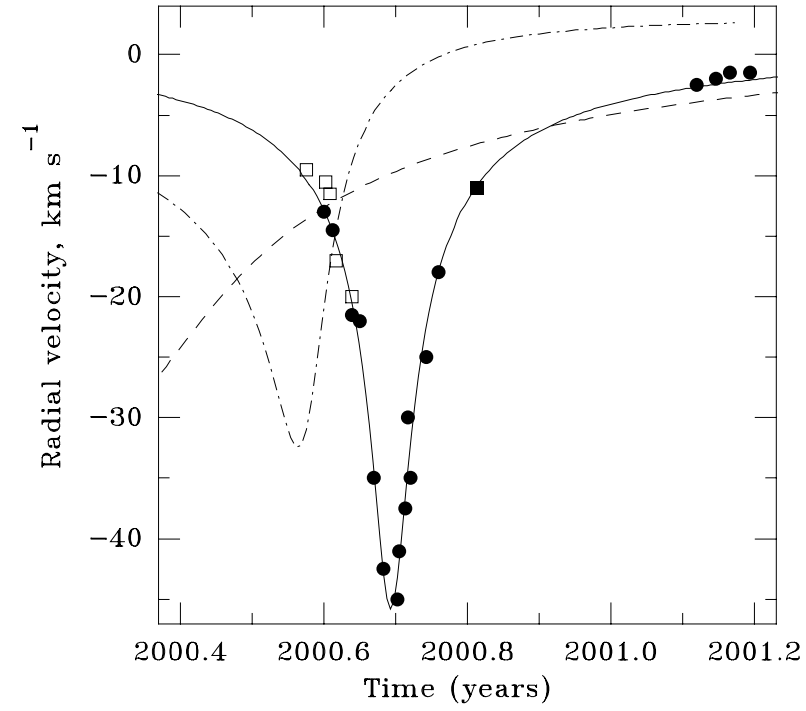

Fig. 6. The mean $R V$ of the $\mathrm{H} \alpha$ profile from the Ritter (filled circles), CAO (open squares), and ESO (filled square) data. The measurements close in time to each other are averaged. Their uncertainties are of the order of the point size. The observational data are shifted by $+6 \mathrm{~km} \mathrm{~s}^{-1}$ to account for the systemic velocity. The line types correspond to those in Fig. 5.

short lived outbursts also occurred (Rivinius et al. 1998). From such comparisons we might differentiate two types of outbursts in Be stars: 1) short outbursts, produced by episodic mass ejection, which give rise to a non-stable circumstellar envelope that dissipates in few weeks; 2) long outbursts, produced by continuous mass ejection, which allow the formation of stable circumstellar envelopes that last several decades. $\mu$ Cen presents both types of outbursts, while $\gamma$ Cas, and so far $\delta$ Sco, present only the long lived ones.

\section{Conclusions}

We have presented and analysed the results of our spectroscopic observations of the $\delta$ Sco binary system during the 2000 periastron passage. The $R V$ curve derived from our $\mathrm{H} \alpha$ line data was used to refine the orbital solution previously derived from published speckle interferometric data. In particular, we determined new values of the periastron epoch and longitude as well as of the orbital inclination angle. The eccentricity value turned out to be close to that in the solution of Hartkopf et al. (1996). The new orbital solution indicates that the binary is detached, and no direct mass transfer takes place. A hypothesis that the primary's nonradial pulsations are amplified at periastron and are responsible for the mass loss is suggested.

Our data, supplemented by the results of visual photometry, suggest a scenario which explains most of the phenomena observed during the 2000/1 $\delta$ Sco observing campaign. For example, the photometric dip centered at periastron and certain changes of the line profiles are attributed to an attenuation of the primary by its disk as 


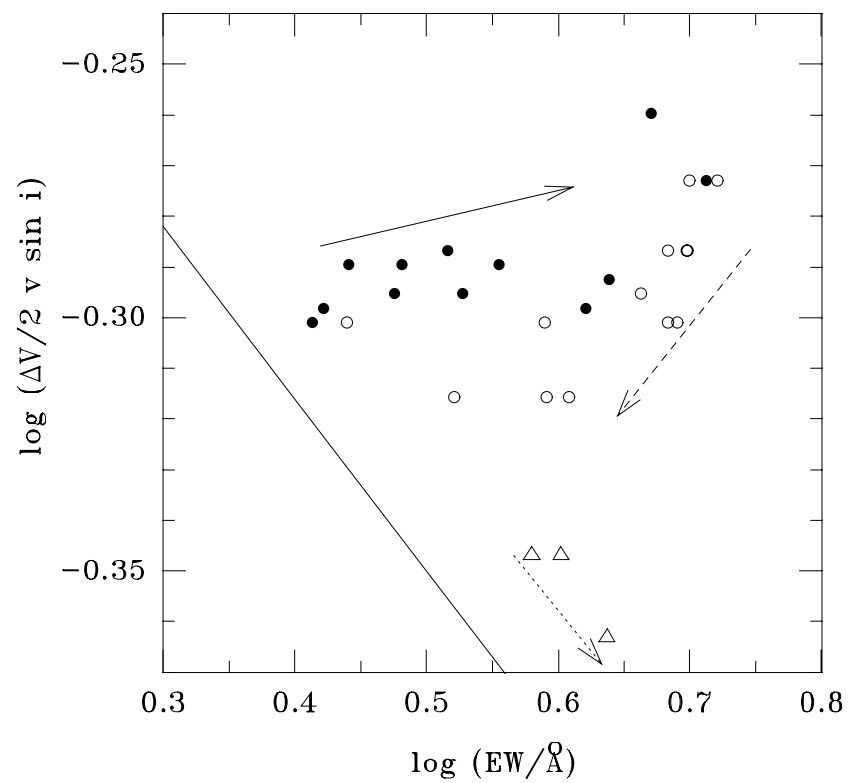

Fig. 7. The relative peak separation of the $\mathrm{H} \alpha$ line versus its $E W$. The data obtained before periastron are shown by filled circles, those obtained after periastron in 2000 by open circles, and the data obtained in 2001 by triangles. The arrow-head lines show temporal trends before (solid) and after (dashed) periastron in 2000, and in 2001 (dotted). The solid line represents the average relationship between the displayed parameters for Be stars found by Hanuschik (1989).

well as to variations of the disk's optical depth, density, and size.

We plan to continue studying this remarkable system. This will include follow up spectroscopic, photometric, and polarimetric observations as well as efforts to model the observed phenomena in more detail.

Acknowledgements. We thank W. J. Fischer and I. Mihailov for their help in obtaining spectra at Ritter. A. M. and K. S. B. acknowledge support from NASA grant NAG5-8054. K. S. B. is a Cottrell Scholar of the Research Corporation, and gratefully acknowledges their support. Support for observational research at Ritter Observatory has been provided by The University of Toledo, by NSF grant AST-9024802 to B. W. Bopp, by an AAS small grant (NASA funding), and by a grant from the Fund for Astrophysical Research. Technical support is provided by R. J. Burmeister. P. R. acknowledges partial support from the European Union Training and Mobility of Researchers Network Grant ERBFMRX/CT98/0195. The G. D. Cassini telescope is operated at the Loiano Observatory by the Osservatorio Astronomico di Bologna. The SAAO spectrum was obtained as part of a different project, which results have not been published yet. I. N. would like to thank D. A. H. Buckley for his help with the observations at SAAO and J. J. Rodes for pre-processing of the data. This research has made use of the SIMBAD database operated at CDS, Strasbourg, France.

\section{References}

Bedding, T. R. 1993, AJ, 106, 768

Blackwell, D. E., Petford, A. D., \& Shallis, M. J. 1980, A\&A, 82,249

Božić, H., Harmanec, P., Horn, J., et al. 1995, A\&A, 304, 235

Brown, A. G. A., \& Verschueren, W. 1997, A\&A, 319, 811

Coté, J., \& van Kerkwijk, M. H. 1993, A\&A, 274, 870

Cowley, A. P., \& Marlborough, J. M. 1968, PASP, 80, 42

Doazan, V., Franco, M., Rusconi, L., Sedmak, G., \& Stalio, R. 1983, A\&A, 128, 171

ESA 1997, The Hipparcos and Tycho Catalogues ESA SP-1200

Evans, D. S. 1967, in Determination of Radial Velocities and Their Applications, ed. A. H. Batten, \& J. F. Heard, Proc. of the IAU Symp. 30 (Academic Press, London), 57

Fabregat, J., Reig, P., \& Otero, S. 2000, IAUC, 7461

Fu, H. H., Hartkopf, W. L., Mason, B. D., et al. 1997, AJ, 114, 1623

Grigsby, J. A., Morrison, N. D., \& Anderson, L. S. 1992, ApJS, 78,205

Hummer, D. G., Abbott, D. C., Voels, S. A., \& Bohannan, B. 1988, ApJ, 328, 704

Hanuschik, R., Kozok, J. R., \& Kaiser, D. 1988, A\&A, 189, 147

Hanuschik, R. 1989, Ap\&SS, 161, 63

Hanuschik, R. W., Dachs, J., Baudzus, M., \& Thimm, G. 1993, A\&A, 274, 356

Harmanec, P. 1998, A\&A, 335, 173

Harmanec, P., Habuda, P., Śtefl, S., et al. 2000, A\&A, 364, L85

Hartkopf, W. L., Mason, B. D., \& McAlister, H. A. 1996, AJ, 111,370

Heasley, J. N., Wolff, S. C., \& Timothy, J. G. 1982, ApJ, 262, 663

Heasley, J. N., \& Wolff, S. C. 1983, ApJ, 269, 634

Hogg, A. R. 1958, Mount Stromlo Obs. Mimeo., No. 2

Hubeny, I., Lanz, T., \& Jeffery, C. S. 1995, Synspec - A User's Guide, Version 36

Innes, R. T. A. 1901, MNRAS, 61, 414

Kallrath, J., \& Milone, E. F. 1998, Eclipsing binary stars. Modeling and Analysis., Springer

Kurucz, R. L. 1994, Smithsonian Astrophys. Obs., CD-ROM No. 19

Leone, F., \& Lanzafame, A. C. 1998, A\&A, 330, 306

Levato, H., Malaroda, S., Morrell, N., \& Solivella, G. 1987, ApJS, 64, 487

McAlister, H. A., \& Hartkopf, W. L. 1988, CHARA contribution, 2

McAlister, H. A., Hartkopf, W. L., \& Franz, O. G. 1988, AJ, 99, 965

Otero, S., Fraiser, B., \& Christopher, L. 2001, IBVS, 5026

Peters, G. J. 1986, ApJ, 301, L61

Quirrenbach, A., Bjorkman, K. S., Bjorkman, J. E., et al. 1997, ApJ, 479, 477

Rivinius, T., Baade, D., Štefl, S., et al. 1998, A\&A, 333, 125

Shortridge, K., Meyerdicks, H., Currie, M., et al. 1997, Starlink User Note, 86.15, R.A.L.

Smith, M. A. 1986, ApJ, 304, 728

Snow, T. P. Jr. 1981, ApJ, 251, 139

van Hoof, A., Bertiau, F. C., \& Deurinck, R. S. J. 1963, ApJ, 137,824 